\title{
Protée
}

\section{Clamence et l'interlocuteur : pragmatique de la manipulation et de l'identification dans le discours de La Chute de Camus}

\section{Gilles Visy}

Volume 32, numéro 2, automne 2004

L’archivage numérique : conditions, enjeux, effets

URI : https://id.erudit.org/iderudit/011177ar

DOI : https://doi.org/10.7202/011177ar

Aller au sommaire du numéro

\section{Éditeur(s)}

Département des arts et lettres - Université du Québec à Chicoutimi

ISSN

0300-3523 (imprimé)

1708-2307 (numérique)

Découvrir la revue

Citer cet article

Visy, G. (2004). Clamence et l'interlocuteur : pragmatique de la manipulation et de l'identification dans le discours de La Chute de Camus. Protée, 32(2), 99-103. https://doi.org/10.7202/011177ar

\section{Résumé de l'article}

Le double jeu de Clamence, à la fois juge et pénitent dans l'interface de l'être et du paraître, semble être le ressort de l'identification du lecteur à l'interlocuteur. Clamence s'adresse à une sorte de grand énonciataire : l'humanité en général et par ricochet le lecteur. La pragmatique du discours de La Chute se construit sur quatre actants : énonciateur-locuteur / interlocuteur-énonciataire. Ces deux entités n'en font qu'une seule et, par contrecoup, l'énonciateur sera assimilé à l'énonciataire par le biais du lecteur. Dans La Chute, émerge en fait tout un jeu de miroirs discursifs dans lequel, d'une part, Clamence élabore son mea culpa et, d'autre part, juge l'humanité comme s'il était une sorte de démiurge divin. Le réquisitoire s'achève sur une note pessimiste qui ne fait que traduire une farce noire dont le procès discursif n'est que pure ironie. 


\title{
CLAMENCE ET L'INTERLOCUTEUR: PRAGMATIQUE DE LA MANIPULATION ET DE L'IDENTIFICATION DANS LE DISCOURS DE LA CHUTE DE CAMUS
}

\author{
GiLles Visy
}

\section{Introduction}

CEuvre grinçante, dont le lecteur ne sait si elle pousse les idées de Camus à la limite, si elle les défigure ou si elle en prend délibérément le contre-pied, La Chute offre le mérite de poser des problèmes littéraires et surtout linguistiques. En lisant ce récit ou ce roman - il semble d'ailleurs que la critique littéraire ait des difficultés à le ranger dans un genre précis ${ }^{1}$-, il est frappant de constater les nombreuses influences littéraires et philosophiques.

Camus connaissait Humain, trop humain de Nietzsche, et ses annotations reposaient sur un «but gigantesque: la connaissance de la vérité» (Camus, 1989: 105). Cette quête devient pour Clamence sa principale préoccupation, qui le conduira au désespoir, vers une voie sans issue. Dès lors, l'influence de Kafka semble couler de source: Camus était imprégné de l'univers absurde du Procès, qui pose le problème de la culpabilité. Dans L'Étranger, Meursault ne peut s'empêcher de dire: «Ce n'est pas de ma faute» (1998: 9).

Cependant, l'influence primordiale reste celle de Dostoïevski; en effet, Camus a trouvé, en lisant Écrits dans un souterrain, l'idée du soliloque avec un interlocuteur muet: "Nous avons remplacé le dialogue par le communiqué" (1997: 42). Tantôt l'homme se parle à lui-même, tantôt il s'adresse à quelque auditeur invisible, à une sorte de juge. En ces termes, le soliloque de La Chute, à l'image d'un rapport intime avec une instance juridique quasi divine, est défini. Clamence se délecte à comparaître devant une entité hypothétique ou pour le moins virtuelle. Il se nomme lui-même «juge-pénitent», humble, il semble prêt à se confesser: "Une chose est simple dans mon cas, je ne possède rien" (ibid.: 13).

L'aveu du protagoniste s'apparente à celui de Nicholas Stavroguine dans Les Possédés ou encore à celui de Temple Stevens dans Requiem pour une none. Lors de l'écriture de La Chute, Camus était sans doute particulièrement sensible à la douleur introspective dans laquelle nous font pénétrer Dostö̈evski et Faulkner. Clamence en témoignera à sa manière: sensible également au problème d'une culpabilité probablement liée à la souffrance humaine, une possible rémission semble s'exercer à travers le discours ${ }^{2}$ de l'aveu. Mais de quel discours s'agit-il? Le double jeu de Clamence, à la fois juge et pénitent dans l'interface de l'être et du paraître, semble être le ressort de l'identification du lecteur à l'interlocuteur. À la fois neutre et ambiguë, l'ambivalence de l'interlocuteur reflète l'effondrement des valeurs linguistiques, idéologiques et religieuses. Quelle stratégie de manipulation utilise Clamence dans l'énonciation? Comment l'interlocuteur se définit à travers une identification protéiforme?

Les manipulations de Clamence dans l'énonciation: «la chute»

Le statut actantiel du locuteur et de l'interlocuteur reste complexe. Il s'agit d'une sorte d'entité, de grand énonciateur qui, par un débrayage, délègue ses pouvoirs au locuteur Clamence. En fait, le «je» est la projection de cette instance énonciatrice suprême à travers le discours de Clamence. Le choix du nom n'est d'ailleurs pas le fruit du hasard. Choisi par l'auteur, reflétant quelque peu l'image de l'in- 
terlocuteur, le nom du protagoniste provient du latin clamans, qui signifie parole. Ce substantif a une connotation religieuse; mais bien loin de servir la gloire du Christ, les connaissances théologiques de Clamence lui permettent plutôt de blasphémer avec raffinement: "Vous parliez du jugement dernier. Permettez-moi d'en rire respectueusement» (Camus, 1997: 94). Le choix du prénom Jean-Baptiste n'est qu'une pure dérision, le locuteur se considérant comme le prophète "clamant» haut et fort sa voix dans le désert: vox clamantis in deserto. Ce saint patron s'était servi de l'eau pour baptiser le Christ et annoncer des temps nouveaux, alors que pour Clamence elle a été l'instrument de sa chute: devant un camarade agonisant, il laissera une femme se noyer, tandis qu'il sera "Pape».

Si Judas, après sa trahison, n'osait plus regarder son maître, Clamence relève la tête et se moque des valeurs judéochrétiennes: "prophète vide pour temps médiocres» (ibid.: 99). Et il a choisi le désert pour crier le temps de la déchéance et la fin de l'humanité: «Trop de gens ont décidé de se passer de la générosité pour pratiquer la charité» (ibid.: 97).

Le discours de Clamence-locuteur repose donc sur des généralités de type proverbial. Elles permettent à JeanBaptiste, grâce à une sorte de "réembrayage», de redevenir le grand énonciateur présupposé. Il existe donc un double jeu entre ces deux actants. Clamence serait une sorte de symbole de la chute, mais également un être humain avec ses faiblesses. La fatigue, la maladie, les moments de dépression caractérisent son rôle de prédicateur. De surcroît, le personnage trahit la misère humaine en montrant au lecteur l'image d'une loque (au lieu de dire cette misère).

Intervient donc le statut supposé de l'interlocuteur. Clamence s'adresse à une sorte de grand énonciataire: l'humanité en général. La pragmatique du discours de La Chute se construit sur quatre actants: énonciateur-locuteur / interlocuteur-énonciataire. Elle se complique avec l'ajout d'un troisième type de relation. Au fur et à mesure de la lecture, le locuteur et l'interlocuteur ont tendance à se confondre. Ces entités n'en font qu'une seule et, par conséquent, l'énonciateur sera assimilé à l'énonciataire. En somme, tout se déroule comme si le premier faisait un retour sur luimême, comme dans un discours introspectif. Cette stratégie discursive se confirme par le rôle déictique des pronoms personnels.

Le «vous», à la première lecture, est bien plus qu'une règle de courtoisie, ce pronom pluriel (étant un substitut de l'humanité) pourrait représenter l'ensemble des interlocuteurs de Clamence, y compris le lecteur. Dans un premier temps, le protagoniste s'adresse à son interlocuteur par le biais du "vous», distribuant ainsi les rôles actantiels sans ambiguïté: vous = Clamence + interlocuteur: «vous et moi seulement» (ibid.: 64).

Ensuite, il utilise des formules plus familières quasi hypochoristiques: «mon cher compatriote» (ibid.: 39), «cher ami» (ibid.: 65). Par un glissement progressif, l'interlocuteur sera associé au locuteur. Ainsi, le «vous» liminaire devient le premier pronom personnel pluriel: «nous nous confions rarement à ceux qui sont meilleurs que nous" (ibid.: 72). Puis «nous», à l'image d'un «je» + «tu» ou d'un «je» + «tu pluriel», se transforme en tournure impersonnelle: «on» = «je» + identité non définie. L'utilisation $\mathrm{du}$ «nous» et du «on" incite le lecteur à identifier Clamence à son interlocuteur: "Alors, insensiblement, je passe, dans mon discours, du "je" au "nous" (ibid.: 117). Le lecteur se projettera sur le premier comme sur le second.

Ce rebondissement provient également d'un jeu pragmatique de questions, de réponses et de réactions de l'interlocuteur. Clamence donne l'impression qu'il intègre dans son propre discours les paroles de son interlocuteur. En réalité, elle sont inexistantes. La passivité de l'interlocuteur favorise les prises de positions idéologiques de Clamence: "chaque homme a besoin d'esclaves comme d'air pur. Commander, c'est respirer, vous êtes bien de cet avis?» (Camus, 1997: 41). Le discours présupposé de l'interlocuteur devient le faire-valoir des propos du locuteur. Ce dernier reste le seul maître de l'énonciation. Il feint de demander l'avis de l'interlocuteur et, en même temps, il tranche la question plus pour la forme que pour obtenir une réponse. C'est un véritable procédé de rhétorique et la question phatique "vous êtes bien de cet avis?», qui impliquerait une réponse directe, sert uniquement à établir le contact avec l'interlocuteur. Le lecteur est porté à croire à l'existence de l'interlocuteur comme si Clamence conversait avec quelqu'un. Il s'agit en fait d'un faux monologue ou d'un dialogue implicite, dans lequel les brèves interventions de l'interlocuteur ne sont connues que dans la mesure où le protagoniste les relève ou les répète sur le mode interrogatif: "N'était-ce pas cela, en effet, l'Éden, cher monsieur: la vie en prise directe?» (Camus, 1997: 27). L'existence de l'interlocuteur n'a de fondement que sur un discours dont la fonction repose sur le "conatif»: 
Il trouve son "existence linguistique», principalement par les interrogations. Encore une fois, est dénoncé le mythe du personnage qui devient ici «lindividu» allocutépar la présente instance $d u$ discours contenant l'instance linguistique TU.

(Reuteur, 1980: 47)

L'auteur fait répéter par son personnage, au besoin avec une certaine emphase, les paroles de son correspondant nécessaires à l'intelligence de la situation de communication. Celle-ci est rendue efficace par l'utilisation du présent de l'indicatif, temps déictique qui actualise le moment de l'énonciation. Dès ses premiers mots, en effet, Clamence, en proposant ses services, impose simultanément sa présence avec le temps présent: «Puis-je monsieur, vous proposer mes services, sans risquer d'être importun?» (Camus, 1997: 7). De surcroît, le texte ne cesse de rappeler, par des adverbes de temps et certaines prépositions ${ }^{3}$, la prégnance de l'instant actuel (voire actualisé): «Vous partez déjà?» (ibid.: 13); "À demain donc» (ibid.: 17); "Voilà, j'ai pignon sur rue, je trafique des esclaves, je vends de la chaire noire» (ibid.: 40-41); «Mais nous sommes arrivés, voici ma maison, mon abri» (ibid.: 62). Cette mise en scène révèle des propos savamment orchestrés: «mon discours est orienté [...], mes délires [...] dirigés» (ibid.: 122). Bien que l'échange discursif soit unilatéral, la manipulation pragmatique repose sur une énonciation implicite de l'interlocuteur: «la communication serait impossible si l'on ne présupposait pas acquis un certain nombre d'informations, à partir desquelles il est possible d'en introduire de nouvelles» (Maingueneau, 1990: 81). Tout repose sur des sous-entendus du locuteur que l'interlocuteur, par le biais du lecteur, décryptera en tant que co-énonciateur. Clamence avance un contenu énonciatif sans en prendre totalement la responsabilité: «Ah! je vous ai intrigué avec cette histoire» (Camus, 1997: 19). Il s'agit d'une sorte de devinette posée à l'interlocuteur.

En réalité, l'efficacité du discours repose sur une bipolarité, l'interlocuteur est à la fois le sujet (en temps que personne) et l'objet (prétexte à la confession fictionnelle) du récit de la chute. Si le discours reste le lieu privilégié du rapport de force entre le locuteur et l'interlocuteur, lui attribuant ainsi une relative autonomie, il n'en reste pas moins un discours piégé. Le langage du locuteur devient «l'arme fatale» de la chute pour celui qui s'y identifierait, sans oublier l'interlocuteur.

\section{L'interlocuteur: identification et ambiguïté}

Si la narration à la première personne est courante, il est moins fréquent que l'auteur d'un roman abandonne en apparence la conduite du récit à son personnage. Le récit de La Chute repose sur cette longue confession de Clamence qui mobilise d'autorité le discours comme si l'auteur feignait de s'en détacher. En réalité, Clamence n'est que «l'individu qui énonce la présente instance de discours contenant l'instance linguistique "je” " (Benveniste, 1966: 252). Dès lors, l'auteur semble être surpassé par son protagoniste qui est plus à même de reconstruire et d'éclairer les événements, les ayant vécus pleinement. Dans La Chute, le discours de Clamence reflète l'univers philosophique de Camus, qui pourtant n'en est que le scripteur. Le protagoniste est le seul qui puisse donner au lecteur les signifiants précis de l'interlocuteur. C'est un avocat d'une quarantaine d'années qui voyage en Hollande. Cultivée, il a lu Dante, la Bible et maîtrise l'imparfait du subjonctif. Au demeurant, il s'agit d'un individu sans épaisseur psychologique, comme certains personnages du nouveau roman. Ce manque de substance psychique favorise l'identification du lecteur à l'interlocuteur. En outre, le discours de Clamence n'a de cesse d'éveiller la curiosité de son interlocuteur et, par contrecoup, celle du lecteur. Joignant la coquetterie au talent, Clamence laisse croire à son compagnon qu'il ne fait que répondre à ses sollicitations. Il «clame» une fausse préciosité: «Je me retire, monsieur, heureux de vous avoir obligé. Je vous remercie et j'accepterais si j'étais sûr de ne pas jouer les fâcheux" (Camus, 1997: 7). Il pique sur le vif l'intérêt de son interloculeur et fait semblant d'y céder: «Vraiment, mon cher compatriote, je vous suis reconnaissant de votre curiosité. Pourtant, mon histoire n'a rien d'extraordinaire. Sachez, puisque vous y tenez [...]” (ibid.: 39). Clamence, fin stratège, le laisse prendre l'initiative du prochain rendezvous après l'avoir séduit amicalement: «Demain? oui, comme vous voudrez» (ibid.: 62). Sans perdre le fil de sa pensée, il feint d'interrompre le discours lorsqu'il voit que son interlocuteur est pendu à ses lèvres: «Voulez-vous que nous nous taisions? Non, je vous intéresse? Vous êtes bien honnête» (ibid.: 82).

Cabotin, Clamence n'attend pas la fin de ses propos pour se féliciter de son talent. Il retarde fréquemment son discours, attendant sans doute une réaction qui, d'ailleurs, ne se produit à aucun moment dans le récit. Cette technique illocutoire s'apparente à celle qui structure l'énonciation 
dans Jacques Le Fataliste et dans Le Neveu de Rameau. Cependant, Diderot voulait que ces retards discursifs servent les dialogues et leur logique dans les réparties, alors que le soliloque de La Chute reproduit un discours à bâtons rompus qui repose sur l'aléatoire. Cette impression de discontinuité est accentuée par l'inversion de l'ordre logique des faits. Clamence évoque d'abord les effets, tels que «le malaise» et "la privation du bonheur", puis relate la cause avec le récit de la noyade. Malgré un monologue faussement décousu et l'art illusoire du comédien qui joue l'aveu calculé, les paroles de Clamence fusent sous l'égide de l'impulsion. Cette volonté de confession relève d'une pathologie de l'effondrement. S'il y a chute des valeurs morales et religieuses, il y a aussi chute de l'individu.

Grâce à cette identification interlocuteur-lecteur, émerge un jeu de miroirs discursifs dans lequel, d'une part, Clamence élabore son mea culpa et, d'autre part, juge l'humanité comme s'il était une sorte de démiurge divin. Ce juge suprême, sans connotation religieuse, est le double de Clamence. Mais sa présence, dans le discours, n'est pas définie, elle reste immatérielle: «Mon image souriait dans la glace, mais il me sembla que mon sourire était double» (ibid.: 37). Le verbe «sembler» exprime une modalité qui repose sur la probabilité, ce qui rend cette présence intangible.

L'interlocuteur est à la fois juge et victime de Clamence, qui se manifeste comme juge-pénitent. À la première lecture, cette confession introspective ressemble à une sorte de psychanalyse. Le transfert énonciatif de Clamence sur l'interlocuteur se construit de la manière suivante: le patient-Clamence veut sans cesse se dérober à son interlocuteur-psychanalyste. Il dénonce ce qui le torture, ce qu’il n'a jamais osé dire auparavant. Mais à la relecture, l'interlocuteur s'avère être une victime. L'appellation «juge-pénitent", et surtout le premier vocable, confirme le statut de dominé de l'interlocuteur. Dans un premier temps, il s'accuse, justifiant ainsi le deuxième vocable, tout en multipliant les nuances et les digressions. Ensuite, il adapte son discours à l'interlocuteur et le conduit à renchérir les fausses maladresses énonciatives. Clamence élargit ainsi ses malheurs à l'ensemble des individus. Voulant être l'arbre qui cache la forêt, il se fait «l'écrivant» de l'expérience de tous, prenant sur lui les faiblesses de chacun. Pour lui, transcripteur et interprète de tout le monde et de personne, la chute devient absurde: "Voilà hélas ce que je suis!» (ibid.: 117). Le réquisitoire s'achève sur une note pessimiste qui ne fait que traduire une farce noire dont le procès n'est que pure ironie: «L'univers entier se mit alors à rire autour de moi» (ibid.: 70).

Le processus d'identification demeure ambigu, car il se fonde également sur le couple locuteur-interlocuteur. Il est vrai que la personnalité de ceux à qui l'on se confesse révèle autant de nous-mêmes que ce qu'on leur confie. À partir de ce prédicat quasi proverbial, tout laisse à penser que Clamence est un double de son interlocuteur. Cependant, l'image floue de ce dernier laisse en suspens un avantage non négligeable, car rien ne prouve, dans le discours de Clamence, que son interlocuteur sombrera dans la déchéance. Néanmoins, le locuteur voudrait bien faire chuter le reste de l'humanité avec lui. Clamence, atteint d'une certaine forme de démence, structure comme un compositeur les éléments d'une symphonie morbide. Il les expose discrètement, ce qui rend le récit subtil et malsain. Ses considérations préliminaires sur l'organisation de la société ou sur les crimes des nazis et des miliciens semblent jetées au hasard. En réalité, elles servent de scène d'exposition au récit pour faire jaillir le furioso final: la chute. Ce drame donne d'emblée une dimension universelle à laquelle le locuteur prétend. À travers celle-ci, il fait le procès de l'humanité en endossant un autre personnage, une nouvelle profession: juge-pénitent. Clamence met ainsi en accusation l'avocat qu'il était et la déchéance qui l'accable. Épave de l'humanité, il tend son discours à l'interlocuteur et, par glissement, à l'ensemble du monde. Égoïste et égocentrique, il tyrannise tout ce qui se trouve à sa portée, y compris le lecteur: «Moi, moi, moi, voilà le refrain de ma chère vie, et qui s'entendait dans tout ce que je disais» (ibid.: 44).

En fin de compte, énonciateur-locuteur, interlocuteurénonciataire, sans oublier le lecteur, représentent peut-être la même entité. Le discours de Clamence est le catalyseur de l'ensemble de ces actants qui jouent la partition de la déchéance humaine par le biais de l'introspection: «Toujours est-il qu'après de longues études sur moi-même, $j$ 'ai mis au jour la duplicité profonde de la créature» (ibid.: 73).

À travers des instances énonciatives et réceptrices qui sont contradictoires, s'établit malgré tout une identification protéiforme. De plus, le parcours discursif de l'identification est circulatoire et nivèle tous les actants, se faisant l'écho, linguistiquement, de l'anéantissement de toute valeur morale. 


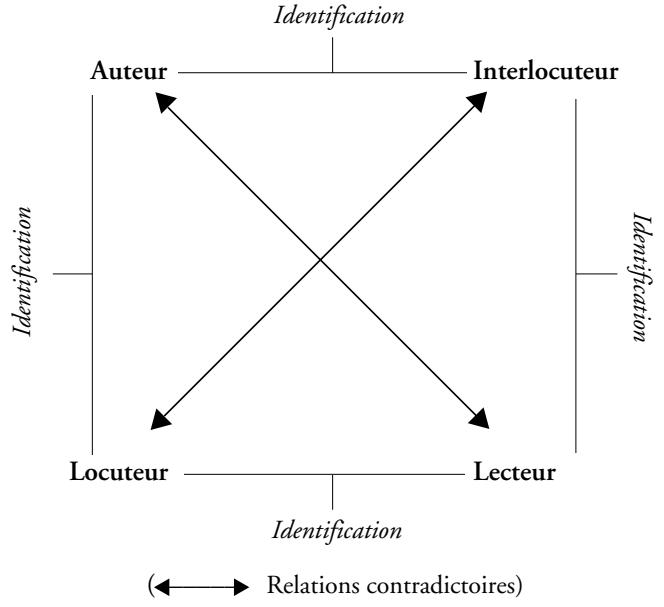

\section{Conclusion}

La voix inaccessible de l'interlocuteur est la métaphore du trajet discursif de Clamence. Si ce dernier est condamné, tout en cherchant une victime pour sa quête introspective, l'interlocuteur est la pure victime qui traduit la déchéance du locuteur. Alors que Clamence prétend s'accuser luimême à travers autrui, sa démarche suicidaire reste le reflet dégradé de sa personne qu'il découvre chez tous les interlocuteurs potentiels: le lecteur au sens large. La culpabilité dans La Chute conduit au désespoir et à l'effondrement des valeurs morales. Clamence est un pessimiste, un nihiliste cynique qui ne respecte plus rien. Qu'est-ce que la chute? "Toute théorie qui refuse l'innocence à l'homme et [...] toute pratique qui le traite en coupable» (ibid.: 111). Ainsi, les perches tendues à cet hypothétique interlocuteur confirment les talents manipulateurs de Clamence. À l'image d'une comédie judiciaire absurde, le locuteur joue sur le plan pragmatique, dans le procès même qu'il met en scène, tous les rôles d'un vrai/faux tribunal: celui de l'accusé, celui de l'avocat de la défense, sans oublier celui de l'accusation; cela explique tous les transferts d'identification. Le tribunal de la "chute» s'est élargi au monde entier avec l'utilisation d'un "vous» de circonspection, de manipulation, qui conduit le lecteur dans l'abîme: «Pour échapper à sa culpabilité, le personnage envisage des stratégies de fuite comme le suicide, l'alcoolisme, la débauche, la dérision généralisée» (Evrand, 1998: 85). La polysémie de «la chute» illustre le corps qui tombe, celui qui est englouti par les eaux. Mais il ne s'agit pas seulement de la déchéance sociale du protagoniste, puisque le signifiant renvoie également au péché originel. Le récit de Camus est une parabole déguisée de la problématique du mal: Adam et Ève chassés du paradis à l'image de Clamence et de son interlocuteur propulsés en enfer.

\section{NOTES}

1. Comme le signale J. Fontanille, le récit conduit «à voir dans tout texte une structure narrative, plus ou moins explicite; elle est explicite dans les genres narratifs, roman, conte, fable ou nouvelle, et implicite dans les autres genres" (1998: 82).

2. Entendu comme " [...] un ensemble de phrases [...], un ensemble de propos organisés; le discours conçu comme le produit de l'énonciation " (Fontanille, 1998: 81).

3. "Voici", "voilà" sont des prépositions qui ont une valeur de verbe pour désigner, la plupart du temps dans l'immédiat, une chose ou une personne. "Voici» les présente sous l'angle de la nouveauté, tandis que "voilà " les caractérise sous l'aspect de ce qui est déjà connu.

\section{RÉFÉRENCES BIBLIOGRAPHIQUES}

BENVENISTE, E. [1966] : «La nature des pronoms », Problèmes de linguistique générale, Paris, Gallimard.

CAmus, A. [1998] : L'Étranger, Paris, Gallimard, coll. "Folio"; [1997] : La Chute, Paris, Gallimard, coll. «Folio»; [1989] : Carnets III, Paris, Gallimard.

Evrand, F. [1998] : Albert Camus, Paris, Ellipses.

FOnTANILle, J. [1998] : Sémiotique du discours, Limoges, PULIM. Maingueneau, D. [1990] : Pragmatique pour le discours littéraire, Paris, Bordas.

Reuteur, Y. [1980]: Textelidéologie dans La Chute de Camus, Paris, Minard. 